\title{
Clinical validation and assessment of aortic hemodynamics using computational fluid dynamics simulations from computed tomography angiography
}

Yulei Zhu ${ }^{1,2}$, Rui Chen ${ }^{1,2}$, Yu-Hsiang Juan ${ }^{3}$, He Li ${ }^{4}$, Jingjing Wang ${ }^{1,2}$, Zhuliang Yu ${ }^{2,5^{*}}$ and Hui Liu i, $^{1 *}$

\section{${ }^{*}$ Correspondence:}

zlyu@scut.edu.cn;

liuhuijiujiu@gmail.com

2 School of Medicine,

South China University

of Technology,

Guangzhou 510006,

Guangdong, China

Full list of author information

is available at the end of the

article

\begin{abstract}
Background: Hemodynamic information including peak systolic pressure (PSP) and peak systolic velocity (PSV) carry an important role in evaluation and diagnosis of congenital heart disease (CHD). Since MDCTA cannot evaluate hemodynamic information directly, the aim of this study is to provide a noninvasive method based on a computational fluid dynamics (CFD) model, derived from multi-detector computed tomography angiography (MDCTA) raw data, to analyze the aortic hemodynamics in infants with $C H D$, and validate these results against echocardiography and cardiac catheter measurements.
\end{abstract}

Methods: This study included 25 patients ( 17 males, and 8 females; a median age of 2 years, range: 4 months -4 years) with CHD. All patients underwent both transthoracic echocardiography (TTE) and MDCTA within 2 weeks prior to cardiac catheterization. CFD models were created from MDCTA raw data. Boundary conditions were confirmed by lumped parameter model and transthoracic echocardiography (TTE). Peak systolic

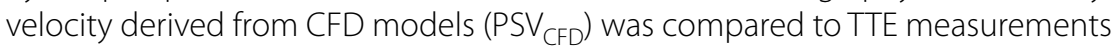
$\left(\right.$ PSV $_{\text {TTE }}$, while the peak systolic pressure derived from CFD (PSP $P_{C F D}$ ) was compared to catheterization ( $P S P_{C C}$ ). Regions with low and high peak systolic wall shear stress (PSWSS) were also evaluated.

Results: PSV CFD $_{\text {and PSP }}$ CFD showed good agreements between PSV ${ }_{\text {TTE }}(r=0.968$, $p<0.001$; mean bias $=-7.68 \mathrm{~cm} / \mathrm{s})$ and $\operatorname{PSP}_{\mathrm{CC}}(r=0.918, p<0.001$; mean bias $=1.405 \mathrm{mmHg}$ ). Regions with low and high PSWSS) can also be visualized. Skewing of velocity or helical blood flow was also observed at aortic arch in patients.

Conclusions: Our result demonstrated that CFD scheme based on MDCTA raw data is an accurate and convenient method in obtaining the velocity and pressure from aorta and displaying the distribution of PSWSS and flow pattern of aorta. The preliminary results from our study demonstrate the capability in combining clinical imaging data and novel CFD tools in infants with CHD and provide a noninvasive approach for diagnose of $\mathrm{CHD}$ such as coarctation of aorta in future.

Keywords: Multi-detector computed tomography angiography, Computational fluid dynamics, Aortic hemodynamics, Congenital heart disease 


\section{Background}

Congenital heart disease (CHD) is a common malformation affecting approximately six per 1000 live births, occurring as an isolated trait or related to multiple congenital anomalies [1]. Despite anatomical evaluation has a great contribution to the diagnosis and treatments of CHD, the hemodynamic evaluation is also indispensable. Peak systolic pressure (PSP) and peak systolic velocity (PSV) were widely used in diagnosis or grading of CHD such as pulmonary arterial hypertension (PAH), aortic valve stenosis (AS), and coarctation of aorta (CoA) [2-4]. An accurate and noninvasive approach to evaluate such hemodynamic information may carry an important role to benefit the clinical diagnosis or grading of CHD.

Cardiac catheterization is the reference standard in obtaining patient-specific hemodynamic analysis, but is associated with patient discomfort and carries potential periprocedural risks [5]. Transthoracic echocardiography (TTE) is the effective first-line technology for obtaining PSV, but is limited in the ability to provide accurate PSP information. Cardiac magnetic resonance (CMR) is another noninvasive method to evaluate hemodynamics in patients [6,7], but it is expensive and more technically demanding. Currently, multi-detector computed tomography angiography (MDCTA) has been widely accepted as an accurate imaging modality to evaluate cardiovascular anatomy for its conveniences, noninvasive procedure and low cost; however, MDCTA cannot provide hemodynamic information directly. Recent studies showed that reliable hemodynamic results of carotid artery, coronary artery and other artery can be acquired using MDCTA raw data alone [8-12], but only few studies had focused on aortic hemodynamic schemes based MDCTA [13].

The aim of this study is to provide a noninvasive method based on a computational fluid dynamics (CFD) model, derived from multi-detector computed tomography angiography (MDCTA) raw data, to analyze the aortic hemodynamics in infants with CHD, and validate these results against echocardiography and cardiac catheter measurements.

\section{Methods}

\section{Study population}

We conducted a single center search of all patients with CHD from July 2015 to October 2016 in our department. Patients were included if they: (1) infants under 4 years old. (2) Had MDCTA and TTE studies, with flow measurement by TTE. (3) Cardiac catheterization within 2 weeks from MDCTA and TTE, with PSP measurement. (4) No malformation and lesion of aorta; Patients were excluded from this study if the studies had poor image quality, or if the above inclusion criteria were not met. There are twenty-five patients (17 males, 8 females) with known CHD were enrolled finally, with a median age of 2 years (range, 4 months -4 years).

\section{Transthoracic echocardiography, MDCTA and cardiac catheterization protocol}

Before undergoing MDCTA, TTE (Philips iE 33 imaging system, Philips Healthcare, Andover, Massachusetts, USA) was performed.

A second-generation dual source CT scanner (Somatom Definition Flash, Siemens Health-care, Forchheim, Germany) was performed using electrocardiographic-gated 
step and shoot protocol. Short-term sedation was achieved with $0.1 \mathrm{mg} / \mathrm{ml}$ of oral chloral hydrate solution. The scans were performed in cranio-caudal direction from the thoracic inlet to the bottom of the heart. MDCTA parameters were as follows: $0.28 \mathrm{~s}$ gantry rotation time, $2 \times 64 \times 0.6 \mathrm{~mm}$ detector collimation, CARE $\mathrm{kV}$ (weight adapted setting for tube voltage and tube current). In the sequential mode, the acquisition window was set at $35-45 \%$ of the $\mathrm{R}-\mathrm{R}$ interval. Safire (strength 3 ) is selected as the iterative reconstruction algorithm, the kernel is $\mathrm{I} 26$, the slice thickness is $0.75 \mathrm{~mm}$, and increment is $0.5 \mathrm{~mm}$. Iodinated contrast medium (Iopamidol, $300 \mathrm{mg} \mathrm{I} / \mathrm{ml}, \mathrm{BRACCO}$, Italy) was injected intravenously at a volume of $1.5-2.0 \mathrm{ml} / \mathrm{kg}$ body weight, followed by $1.0 \mathrm{ml} / \mathrm{kg}$ body weight saline chaser with injection rate $1-2 \mathrm{ml} / \mathrm{s}$. The acquisition delay was determined by the time of contrast medium entering both ventricles.

CC was performed by using Philips Allura Xper FD10 system (Philips Medical Systems, Best, the Netherlands) to get hemodynamics including PSP of the aortic isthmus (AI).

\section{Construction of aorta and mesh generation}

Computational representations of the aorta were created using Mimics 17 (Materialise, Leuven, Belgium) software that facilitates volume visualization and conversion of the MDCTA raw data into geometrically representative computer models, as demonstrated in Fig. 1. Models were discretized using an automatic mesh generation software (Ansys ICEM 14.5, ANSYS, Inc., Canonsburg, Pennsylvania, USA). The mesh generation followed a custom standard protocol with unstructured, formatted tetrahedral. In addition, prism mesh was added to the wall boundary (height $=1$, ratio $=1.2$, numbers $=3$ ) in order to improve the accuracy of hemodynamic parameters adjacent to aortic wall. The total elements in each mesh ranging from 1,000,000 to 2,000,000.

\section{Boundary conditions}

Inflow and outflow boundaries were defined in Fig. 1, and the flow domain was defined as cavity of the reconstructed geometry. Velocity information in TTE data was mapped to the inlet of CFD models, while the blood pressure (BP) was used to prescribe the outlet boundaries. Lumped parameter model (LPM) was applied to confirm the outflow boundary conditions if BP of outlet was inaccessible. The parameter of LPM was shown in Table 1, and the schematic illustration of LPM was shown in Fig. 2. The unknown pressure $(P)$ of outlet for CFD models was calculated from LPM, the equation of LPM was given as follow:

$$
P=\left(R_{1}+R_{2}\right) Q-R_{2} C \frac{d P}{d t}+R_{1} R_{2} \frac{d Q}{d t}
$$

where Q was the volume flow rate through brachiocephalic artery (BA), left common carotid artery (LCCA), left subclavian artery (LSA), or descending aorta (DAo) respectively. R1 was the characteristic resistance of the artery, while R2 was Peripheral impedance of the artery, and $C$ was the compliance of artery. The value of $R_{1}, R_{2}$, $C$ were calculated according to previous work $[13,14]$. The derivative items can be calculated using the backward Euler method: 


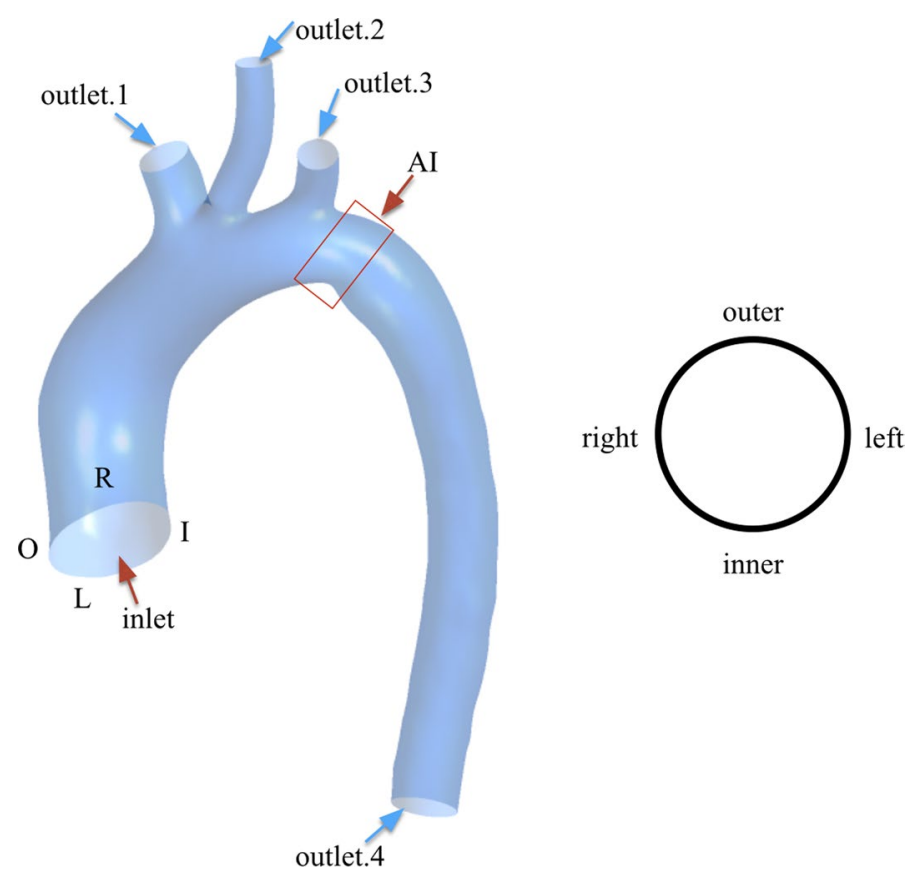

Fig. 1 The patient-specific aortic geometry. An inlet boundary and four outlet boundaries were defined in left image. $O, R, L$ and I represented the four partition of the aortic wall demonstrated in right image. The region identified by 'Al' was aortic isthmus

Table 1 Parameter of LPMs

\begin{tabular}{llll}
\hline Artery & $\mathbf{R}_{\mathbf{1}}(\mathbf{m m H g ~ s} / \mathbf{m l})$ & $\mathbf{R}_{\mathbf{2}}(\mathbf{m m H g ~ s} / \mathbf{m l})$ & $\mathbf{C}(\mathbf{m l} / \mathbf{m m H g})$ \\
\hline BA & 0.100 & 2.480 & 0.466 \\
LCCA & 0.110 & 2.510 & 0.443 \\
LSA & 0.150 & 2.624 & 0.437 \\
DAO & 0.120 & 2.118 & 0.421 \\
\hline
\end{tabular}

BA (brachiocephalic artery), LCCA (left common carotid artery), LSA (left subclavian artery), DAo (descending aorta). $R_{1}$ was characteristic resistance, $R_{2}$ was Peripheral impedance, and $C$ was compliance of artery

$$
\begin{aligned}
& P_{n+1}=\frac{\left(R_{1}+R_{2}+R_{1} \beta\right)-R_{1} \beta Q_{n}+\beta P_{n}}{1+\beta} \\
& \beta=R_{2} C / \Delta t
\end{aligned}
$$

where $\Delta t$ was time interval between $\mathrm{P}_{\mathrm{n}}$ and $\mathrm{P}_{\mathrm{n}+1}$.

\section{Computational fluid dynamics simulation}

Simulation was performed by using Ansys FLUENT 14.5 (Fluent Inc., Lebanon, New Hamshire, USA). In our calculation, there were some assumptions of physical properties of blood according to previous work: (1) Blood was assumed to be incompressible, viscous, Newtonian fluid. (2) The density $(\rho)$ and viscosity $(\mu)$ of blood was 


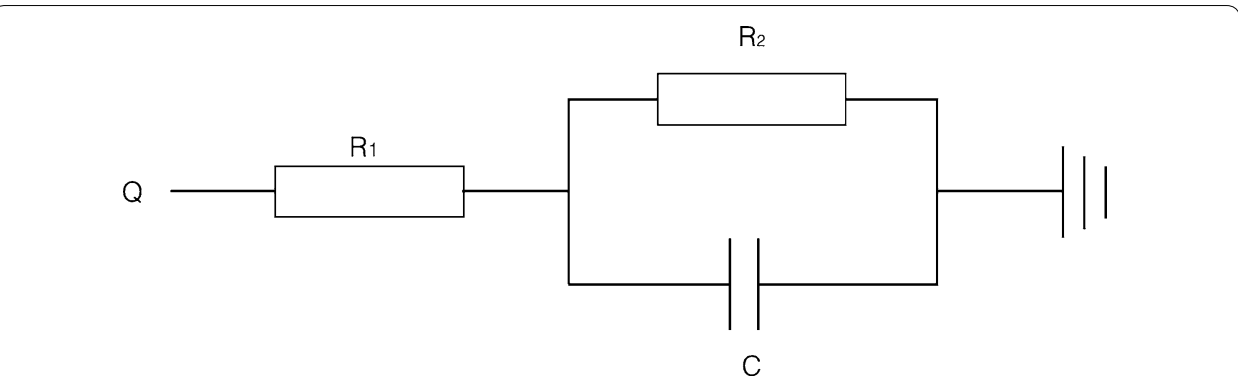

Fig. 2 Scheme of $L P M . R_{1}$ represented the characteristic resistance, $R_{2}$ represented the peripheral impedance, and $C$ represented the compliance of artery. $Q$ was flow rate of artery, and pressure on outlet was calculated by solving the differential equation

assumed to be constant and equals to $1050 \mathrm{~kg} / \mathrm{m}^{3}$ and $0.004 \mathrm{~Pa} \mathrm{~s}$ [14-17]. Calculation solved the Navier-Stokes equations as follow [18]:

$$
\begin{aligned}
& \rho\left(\frac{d u}{d t}+u \bullet \nabla u\right)=-\nabla P+\mu \nabla^{2}+F \\
& -\nabla \bullet u=0
\end{aligned}
$$

where $u$ was the blood velocity, $F$ was body force equals to zero. Fluid structure interaction simulation was wildly used to illustrate the interaction between blood and vessels [19-21]. Since the hemodynamics of the blood is the main research topic in this current study, we have not included fluid structure interaction simulation into this study. Similar approaches had been applied in other related studies to acquire accurate hemodynamics from simulation without FSI [14, 22-24].

\section{Statistical analysis}

The statistical analysis was performed by SPSS (SPSS 22, SPSS Inc., Chicago, USA). For continuous variables, data are expressed as the mean \pm standard deviation $(\mathrm{M} \pm \mathrm{SD})$. All tests were two-sided, and effects were considered significant at $\mathrm{p}<0.05$. Normality was tested using the Kolmogorov-Smirnov method, and variance homogeneity was then tested with the Levene's test. Group differences were assessed by paired Student's t test in normally distributed (Kolmogorov-Smirnov test) data. Otherwise, the paired Wilcoxon test was used.

To illustrate the accuracy of aorta reconstruction, morphometric parameters (diameters at three locations: aorta ascending, aorta descending, and stenosis) were analyzed by paired Student's $t$-test. To demonstrate the accuracy of our simulation, the agreements between PSV $_{\mathrm{CFD}}$ and $\mathrm{PSV}_{\mathrm{TTE}}, \mathrm{PSP}_{\mathrm{CFD}}$ and $\mathrm{PSP}_{\mathrm{CC}}$ were characterized with the BlandAltman plot and linear fitting. 
Table 2 Calculated and measured geometric parameters

\begin{tabular}{|c|c|c|c|c|c|c|c|c|c|}
\hline \multirow[t]{2}{*}{ Case } & \multicolumn{3}{|c|}{ AscAo } & \multicolumn{3}{|l|}{ Al } & \multicolumn{3}{|l|}{ DAo } \\
\hline & $\mathbf{R}$ & M & $P$ & $\mathbf{R}$ & $M$ & $P$ & $\mathbf{R}$ & $M$ & $P$ \\
\hline 01 & 11.2 & 11.0 & 0.300 & 8.90 & 8.8 & 0.805 & 8.1 & 8.0 & 0.18 \\
\hline 02 & 20.0 & 20.4 & & 13.80 & 13.1 & & 11.5 & 11.7 & \\
\hline 03 & 25.8 & 25.0 & & 19.34 & 19.0 & & 16.1 & 15.0 & \\
\hline 04 & 16.2 & 16.5 & & 10.60 & 11.0 & & 9.1 & 9.7 & \\
\hline 05 & 28.9 & 29.5 & & 17.40 & 17.5 & & 14.5 & 14.5 & \\
\hline 06 & 15.2 & 15.5 & & 10.40 & 11.0 & & 9.1 & 9.4 & \\
\hline 07 & 21.2 & 21.5 & & 13.00 & 13.2 & & 11.8 & 11.4 & \\
\hline 08 & 17.9 & 18.5 & & 11.30 & 11.0 & & 9.8 & 10.0 & \\
\hline 09 & 17.7 & 18.2 & & 12.80 & 13.0 & & 12.6 & 12.0 & \\
\hline 10 & 11.7 & 12.0 & & 8.20 & 8.4 & & 8.0 & 7.8 & \\
\hline 11 & 19.4 & 20.5 & & 10.80 & 11.0 & & 10.0 & 10.0 & \\
\hline 12 & 17.2 & 17.4 & & 12.80 & 12.0 & & 11.4 & 11.5 & \\
\hline 13 & 17.1 & 17.0 & & 14.24 & 14.5 & & 12.3 & 12.0 & \\
\hline 14 & 21.0 & 21.4 & & 16.40 & 16.7 & & 15.3 & 16.0 & \\
\hline 15 & 18.0 & 18.4 & & 9.70 & 10.7 & & 8.9 & 8.4 & \\
\hline 16 & 35.0 & 35.0 & & 13.38 & 13.5 & & 10.0 & 10.0 & \\
\hline 17 & 11.1 & 11.0 & & 7.80 & 6.9 & & 7.0 & 7.7 & \\
\hline 18 & 22.3 & 22.5 & & 15.40 & 15.5 & & 14.1 & 14.0 & \\
\hline 19 & 26.7 & 26.5 & & 13.40 & 12.8 & & 10.7 & 10.3 & \\
\hline 20 & 23.0 & 23.0 & & 13.50 & 13.2 & & 11.0 & 10.2 & \\
\hline 21 & 26.1 & 26.4 & & 17.20 & 17.4 & & 16.0 & 15.4 & \\
\hline 22 & 13.0 & 12.5 & & 6.60 & 6.7 & & 8.3 & 8.7 & \\
\hline 23 & 11.6 & 11.0 & & 5.30 & 5.5 & & 8.4 & 8.0 & \\
\hline 24 & 17.7 & 17.3 & & 11.80 & 11.5 & & 9.7 & 9.2 & \\
\hline 25 & 16.2 & 15.6 & & 10.10 & 9.7 & & 8.6 & 8.2 & \\
\hline
\end{tabular}

$\mathrm{R}, \mathrm{M}$, and $\mathrm{P}$ were represented the reconstructed aorta, measured aorta and $\mathrm{p}$ value

\section{Results}

Table 2 summarizes the reconstructed and measured geometric parameters of the aorta. Mean reconstructed diameters of the ascending aorta (inlet), descending aorta (outlet.4) and AI were 19. $2 \pm 6.0 \mathrm{~mm}, 10.9 \pm 2.6 \mathrm{~mm}$, and $12.1 \pm 3.5 \mathrm{~mm}$ respectively. The paired $t$ test found no difference between measured diameters and simulated diameters.

The data on PSP $_{\mathrm{CC}}, \mathrm{PSV}_{\mathrm{TTE}}, \mathrm{PSP}_{\mathrm{CFD}}$, and $\mathrm{PSV}_{\mathrm{CFD}}$ are given in Table 3. $\mathrm{PSP}_{\mathrm{CFD}}$ had an excellent correlation (Fig. 3a) with $\operatorname{PSP}_{\mathrm{CC}}(\mathrm{r}=0.918, \mathrm{p}<0.001)$. The mean $\mathrm{PSP}_{\mathrm{CC}}$ was $105.08 \pm 15.38 \mathrm{mmHg}$, while mean $\mathrm{PSP}_{\mathrm{CFD}}$ was $106.48 \pm 15 \mathrm{mmHg}$. The mean bias was $1.405 \mathrm{mmHg}$ (Fig. 3b, 95\% confidence interval -7.237-10.04). PSV $\mathrm{CFD}_{\text {D }}$ was also excellently correlated with PSV $_{\text {TTE }}$ (Fig. $4 \mathrm{a}, \mathrm{r}=0.968, \mathrm{p}<0.001$ ). The mean PSV $\mathrm{PTE}_{\text {TTE }}$ was $152.92 \pm 64.36 \mathrm{~cm} / \mathrm{s}$, while mean $\mathrm{PSV}_{\mathrm{CFD}}$ was $145.24 \pm 61.68 \mathrm{~cm} / \mathrm{s}$. The mean bias was $-7.68 \mathrm{~cm} / \mathrm{s}$ (Fig. 4b, 95\% confidence interval -30.41 to 15.05 ).

During peak systole, on the one hand, lowest PSWSS $(2.83 \pm 1.23 \mathrm{~Pa})$ was observed at right wall of ascending aorta (AscAo) and inner wall of the attachment of arterial ductus ligament adjacent to AI. On the other hand, highest PSWSS $(16.26 \pm 3.43 \mathrm{~Pa})$ was localized at outer wall of aortic arch, ostium of BA (Fig. 5). Streamline was also visualized and demonstrated in Fig. 5, velocity skewed toward the inner wall of AscAo and the region 
Table 3 Measured and simulated PSV and PSP

\begin{tabular}{|c|c|c|c|c|c|c|}
\hline \multirow[t]{2}{*}{ Case } & \multicolumn{3}{|c|}{ PSV (cm/s) } & \multicolumn{3}{|c|}{ PSP $(\mathrm{mmHg})$} \\
\hline & TTE & CFD & $\mathrm{R}^{2}$ & $\overline{C C}$ & CFD & $\mathrm{R}^{2}$ \\
\hline 01 & 160 & 151 & 0.968 & 94 & 92 & 0.918 \\
\hline 02 & 85 & 100 & & 102 & 96 & \\
\hline 03 & 100 & 80 & & 123 & 120 & \\
\hline 04 & 245 & 231 & & 89 & 92 & \\
\hline 05 & 130 & 130 & & 133 & 137 & \\
\hline 06 & 100 & 92 & & 101 & 103 & \\
\hline 07 & 120 & 112 & & 121 & 126 & \\
\hline 08 & 121 & 100 & & 91 & 95 & \\
\hline 09 & 240 & 226 & & 92 & 98 & \\
\hline 10 & 100 & 90 & & 80 & 86 & \\
\hline 11 & 100 & 80 & & 88 & 93 & \\
\hline 12 & 97 & 81 & & 78 & 82 & \\
\hline 13 & 110 & 111 & & 118 & 114 & \\
\hline 14 & 150 & 142 & & 110 & 106 & \\
\hline 15 & 131 & 120 & & 110 & 114 & \\
\hline 16 & 235 & 248 & & 115 & 122 & \\
\hline 17 & 150 & 145 & & 81 & 84 & \\
\hline 18 & 110 & 132 & & 110 & 115 & \\
\hline 19 & 75 & 76 & & 115 & 110 & \\
\hline 20 & 70 & 71 & & 113 & 114 & \\
\hline 21 & 212 & 200 & & 117 & 121 & \\
\hline 22 & 251 & 231 & & 100 & 97 & \\
\hline 23 & 250 & 227 & & 110 & 102 & \\
\hline 24 & 241 & 227 & & 130 & 131 & \\
\hline 25 & 240 & 228 & & 106 & 112 & \\
\hline
\end{tabular}

PSV $_{\text {TTE }}$ and PSV CFD $_{\text {were PSV measured by TTE and calculated by simulation respectively, while PSP }}$ wC and PSP CFD $_{\text {Were PSP }}$ measured by CC and calculated by simulation
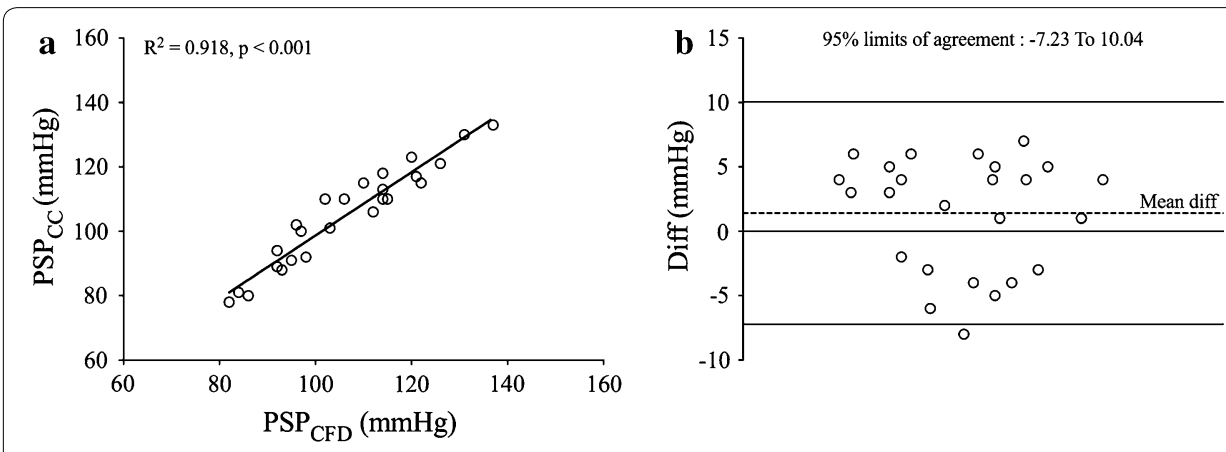

Fig. 3 Validation of PSP derived from CFD. Demonstration of PSV and PSP validation. The image a was linear fitting of PSP, and image $\mathbf{b}$ was Bland-Altman plot of PSP. The reference line of Bland-Altman plots was mean difference $\pm 1.96 * S D$ 

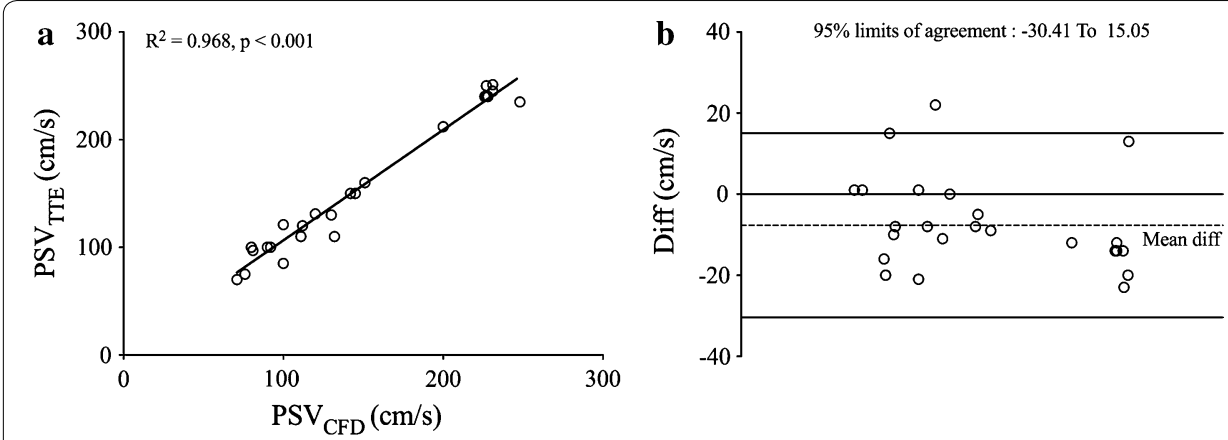

Fig. 4 Validation of PSV derived from CFD. Demonstration of PSV and PSP validation. The image a was linear fitting of PSV, and image $\mathbf{b}$ was Bland-Altman plot of PSV. The reference line of Bland-Altman plots was mean difference $\pm 1.96 * \mathrm{SD}$

between inner and right wall of DAo (Fig. 5). Helical flow was observed at arch (Fig. 5), and there was highest velocity observed at ostium of BA (Fig. 5).

\section{Discussion}

This study compared $\mathrm{PSV}_{\mathrm{CFD}}$ with $\mathrm{PSV}_{\mathrm{TTE}}$ and $\mathrm{PSP}_{\mathrm{CFD}}$ with $\mathrm{PSP}_{\mathrm{CC}}$ respectively. We demonstrated good agreements between reference standard method and CFD method.

Concerning the findings, there are additional factors that must be considered. Obviously, diameters of geometry reconstructed from MDCTA raw data will affect the CFD results, however, paired $t$-test found no difference between measured and reconstructed diameters, it means that geometry constructed from MDCTA raw data was identical to the real anatomy.

Given the results in the previous section, we observed that there are good agreements between PSV $_{\mathrm{TTE}}$ versus PSV $\mathrm{PFD}_{\mathrm{CF}}$ and $\mathrm{PSP}_{\mathrm{CC}}$ versus $\mathrm{PSP}_{\mathrm{CFD}}$. The result demonstrated that CFD method was accurate comparing to TTE and cardiac catheterization. The biases of PSV and PSP were small, and there are many factors contributed to the biases. Data acquisition with MDCTA and catheterization was performed sequentially and thus there could be slight day-to-day variations in stroke volumes and other hemodynamics parameters [6]. More importantly, PSP was measured in sedated patients, whereas MDCTA and TTE were measured when patients awake. But according to the results, the biases were acceptable.

During MDCTA processing, there is a level of uncertainty when reconstructing the 3D aorta geometry based on 2D MDCTA raw data. Precise reconstruction is critical for the outcome of CFD simulation. However, no significant differences between measured and reconstructed geometric parameters were observed in this study.

The step of simulation may also be a factor contributing to the biases. Lumped parameter model was widely used to implement the pressure at outlet boundary according to previous studies [9, 14, 18, 25-27]. However, modeling hemodynamics in aorta with LPM involves a challenging set of constraints. In addition, previous studies have found that no-slip wall boundary is suitable and it is wildly used in vessel modeling. However, accurate wall boundary definition is still an active topic of investigation $[28,29]$. In this work, second order no-slip wall boundary was included in 


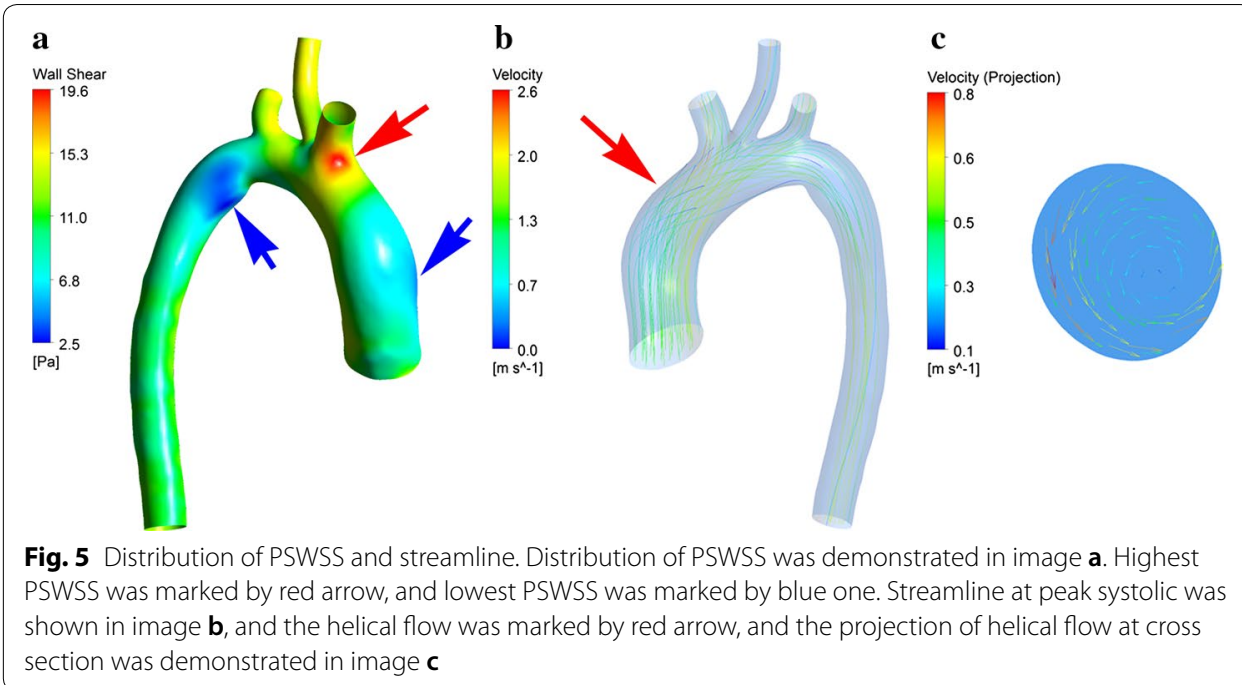

the simulation, with the rational to reduce the computational effort and complexity of simulation.

In most of cases, low PSWSS of aorta was localized at outer or right luminal surface of ascending aorta or inner wall of the attachment of arterial ductus ligament adjacent to AI, while high PSWSS of aorta was located at outer wall of aortic arch, ostium of BA. This result corresponds to the distribution of WSS reported in other studies [14, 24]. In the current study, low PSWSS was observed at outer or right wall of AscAo maybe explained by the skewing velocity profile towards the inner wall during peak systole, while the velocity imposed to outer wall of AscAo was low. On the contrary, the highest PSWSS observed on outer wall of aortic arch and ostium of BA. This maybe explained by the arc shape of aortic arch. In this study, PSWSS on inner wall was lower than PSWSS on AI, and the larger diameter of arterial ductus ligament attachment as compared to AI maybe a factor contributed to this phenomenon.

Study conducted by Chiu et al. proved that PSWSS played an important role for regulating the arrangement and function of endotheliocyte [30]. Previous study also suggests that region with low PSWSS was correlated with areas of atherosclerotic plaque [31], and other study also indicated that excessive PSWSS was related with aneurysm formation of endothelial cells [32]. In addition, PSWSS also played an important role in the formation of aortic dissection (AD). Thubrikar reported that elevated PSWSS was correlated with sites of intimal tears [33], Wen also reported that initial location of tears was coincident with the region of maximal WSS [34], and Nordon reported that low PSWSS was benefit for minimizing the propagation of the dissection [35]. These studies indicated that PSWSS was related to the formation and prognosis of AD. Hence, WSS derived from the MDCTA raw data may provide not only anatomic information on aorta, but also additional information on plaque and dissection development.

Uniform distribution and smooth laminar blood flow was observed at AscAo, while skewing and helical flow was observed at aorta during peak systole. The flow pattern in current study was consistent with other studies [36, 37]. The inertial force was higher than viscous force during peak systolic, which help the development of helical flow, a 
usual physiological phenomenon in our cardiovascular system [38]. Helical flow in aorta exhibits important hemodynamic effect in increasing flux of oxygen [39] and achieving normal level of PSWSS [40]. Moreover, helical blood flow hinders the development of atherosclerotic plaques [23]. Similarly, the luminal surface low-density lipoprotein concentration in the aortic arch can also be reduced by helical flow [41]. Flow pattern generated by CFD based on MDCTA raw data may provide a reliable and convenient method to evaluate flow information.

\section{Study limitations}

There were some limitations in the current study. First, the aortic valve morphology was not considered for its influence on the velocity profile. However, many studies have proven that the influence of valve morphology on velocity was small, and accurate simulation results had been achieved without considering aortic valvular morphology [14, 24, 42, 43]. Second, Constant LPM parameters were used to implement CFD in order to simplify the process of calculation despite unequal aortic morphology in each patient. However, their validated results suggested that the simulation error was negligible compared to other study $[44,45]$. Third, this study had a limited number of subjects recruited in our study, and a follow up study with a larger number of subjects may provide a better valid assessment of aortic hemodynamic from MDCTA.

\section{Conclusions}

Our results showed that CFD scheme based on MDCTA raw data is an accurate and convenient method in obtaining flow velocity and pressure from aorta and displaying the distribution of WSS and flow pattern of aorta. The preliminary results from our study demonstrated the capability in combining clinical imaging data and novel CFD tools in infants with $\mathrm{CHD}$ and provide t provide another noninvasive approach for diagnose of CHD such as CoA, AS or PAH in future.

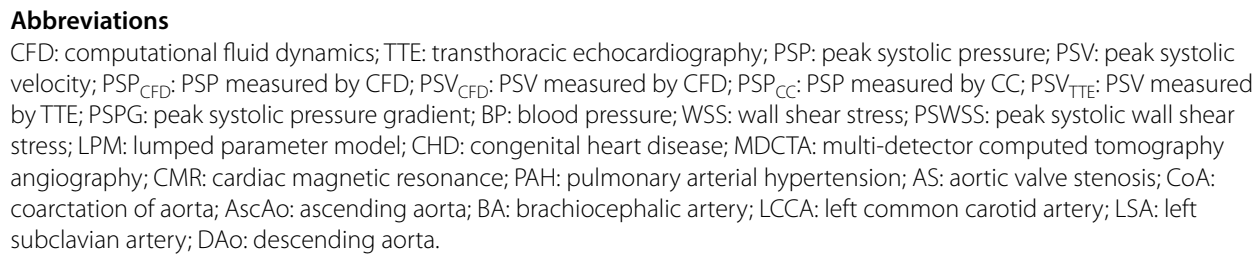
velocity; PSP CFD: $_{\text {PSP measured by CFD; PSV }}$ CFD: PSV measured by CFD; PSP $C$ : PSP measured by CC; PSV TTE: PSV measured by TTE; PSPG: peak systolic pressure gradient; BP: blood pressure; WSS: wall shear stress; PSWSS: peak systolic wall shear stress; LPM: lumped parameter model; CHD: congenital heart disease; MDCTA: multi-detector computed tomography angiography; CMR: cardiac magnetic resonance; PAH: pulmonary arterial hypertension; AS: aortic valve stenosis; CoA: coarctation of aorta; AsCAo: ascending aorta; BA: brachiocephalic artery; LCCA: left common carotid artery; LSA: left subclavian artery; DAo: descending aorta.

\section{Authors' contributions}

$Y Z$ contributed in the research design and drafting of the manuscript. $\mathrm{HL}$ and $\mathrm{ZY}$ supervised and coordinated the study and also contributed in designing of research. $Y Z$ and RC acquired patient clinical information. $Y J$ contributed the editing manuscript and tables. HL contributed in statistical analysis. YZ, RC, and JW contributed to the image acquisition, image reformation, interpretation, preparation of the figures and figure legends. All authors read and approved the final manuscript.

\section{Author details}

'Department of Radiology, Guangdong General Hospital, Guangdong Academy of Medical Sciences, No. 106, Zhong Shan Er Lu, Guangzhou 510080, Guangdong, China. ${ }^{2}$ School of Medicine, South China University of Technology, Guangzhou 510006, Guangdong, China. ${ }^{3}$ Department of Medical Imaging and Intervention, Chang Gung Memorial Hospital, Linkou Chang Gung University, Taoyuan, Taiwan. ${ }^{4}$ Department of Cardiac Surgery, Guangdong Cardiovascular Institute, Guangdong Provincial Key Laboratory of South China Structural Heart Disease, Guangdong General Hospital, Guangdong Academy of Medical Sciences, No. 106, Zhong Shan Er Lu, Guangzhou 510080, Guangdong, China. ${ }^{5}$ College of Automation Science and Technology, South China University of Technology, 381 Wushan Road, Guangzhou 510080, Guangdong, China. 


\section{Acknowledgements}

None.

\section{Competing interests}

The authors declared that they have no competing interests.

\section{Availability of data and materials}

The datasets used or analyzed during the current study are available from the corresponding author on reasonable request.

\section{Consent for publication}

All participates had been informed before submission, and all authors agreed the publication.

\section{Ethic approval and consent to participate}

The study was approved by the institutional Research Ethics Committee following the ethical guidelines of the 1975 Declaration of Helsinki. Informed consent was obtained from all individual participants included in the study.

\section{Funding}

The current study was funded by national natural science foundation of China (No. 81771799).

\section{Publisher's Note}

Springer Nature remains neutral with regard to jurisdictional claims in published maps and institutional affiliations.

Received: 28 August 2017 Accepted: 23 April 2018

Published online: 02 May 2018

\section{References}

1. Molck MC, Simioni M, Vieira TP, Sgardioli IC, Monteiro FP, Souza J, Fett-Conte AC, Felix TM, Monlleo IL, Gil-da-Silva-Lopes VL. Genomic imbalances in syndromic congenital heart disease. Jornal de pediatria. 2017;93(5):497-507.

2. Kitabatake A, Inoue M, Asao M, Masuyama T, Tanouchi J, Morita T, Mishima M, Uematsu M, Shimazu T, Hori M, Abe H. Noninvasive evaluation of pulmonary hypertension by a pulsed Doppler technique. Circulation. 1983;68(2):302-9.

3. Suradi H, Hijazi ZM. Current management of coarctation of the aorta. Glob Cardiol Sci Pract. 2015;2015(4):44.

4. Otto CM, Burwash IG, Legget ME, Munt BI, Fujioka M, Healy NL, Kraft CD, Miyake-Hull CY, Schwaegler RG. Prospective study of asymptomatic valvular aortic stenosis. Clinical, echocardiographic, and exercise predictors of outcome. Circulation. 1997;95(9):2262-70.

5. Di Biase L, Burkhardt JD, Mohanty P, Sanchez J, Horton R, Gallinghouse GJ, Lakkireddy D, Verma A, Khaykin Y, Hongo $\mathrm{R}$, et al. Periprocedural stroke and management of major bleeding complications in patients undergoing catheter ablation of atrial fibrillation: the impact of periprocedural therapeutic international normalized ratio. Circulation. 2010;121(23):2550-6.

6. Goubergrits L, Riesenkampff E, Yevtushenko P, Schaller J, Kertzscher U, Hennemuth A, Berger F, Schubert S, Kuehne T. MRI-based computational fluid dynamics for diagnosis and treatment prediction: clinical validation study in patients with coarctation of aorta. J Magn Reson Imaging JMRI. 2015;41(4):909-16.

7. Wong KKL, Kelso RM, Worthley SG, Sanders P, Mazumdar J, Abbott D. Medical imaging and processing methods for cardiac flow reconstruction. J Mech Med Biol. 2009;9(1):1-20.

8. Koo BK, Erglis A, Doh JH, Daniels DV, Jegere S, Kim HS, Dunning A, DeFrance T, Lansky A, Leipsic J, Min JK. Diagnosis of ischemia-causing coronary stenoses by noninvasive fractional flow reserve computed from coronary computed tomographic angiograms. Results from the prospective multicenter DISCOVER-FLOW (diagnosis of ischemia-causing stenoses obtained via noninvasive fractional flow reserve) study. J Am Coll Cardiol. 2011;58(19):1989-97.

9. Liu X, Gao Z, Xiong H, Ghista D, Ren L, Zhang H, Wu W, Huang W, Hau WK. Three-dimensional hemodynamics analysis of the circle of Willis in the patient-specific nonintegral arterial structures. Biomech Model Mechanobiol. 2016;15(6):1439-56.

10. Qin Y, Wu J, Hu Q, Ghista DN, Wong KK. Computational evaluation of smoothed particle hydrodynamics for implementing blood flow modelling through CT reconstructed arteries. J X ray Sci Technol. 2017;25(2):213-32.

11. Wong KKL, Thavornpattanapong P, Cheung SCP, Tu JY. Biomechanical investigation of pulsatile flow in a threedimensional atherosclerotic carotid bifurcation model. J Mech Med Biol. 2013;13(1):1350001.

12. Wong KK, Kelso RM, Worthley SG, Sanders P, Mazumdar J, Abbott D. Cardiac flow analysis applied to phase contrast magnetic resonance imaging of the heart. Ann Biomed Eng. 2009;37(8):1495-515.

13. Nie $P$, Wang $X$, Cheng Z, Ji $X$, Duan $Y$, Chen J. Accuracy, image quality and radiation dose comparison of high-pitch spiral and sequential acquisition on 128-slice dual-source CT angiography in children with congenital heart disease. Eur Radiol. 2012;22(10):2057-66.

14. Menon A, Wendell DC, Wang H, Eddinger TJ, Toth JM, Dholakia RJ, Larsen PM, Jensen ES, LaDisa JF. A coupled experimental and computational approach to quantify deleterious hemodynamics, vascular alterations, and mechanisms of long-term morbidity in response to aortic coarctation. J Pharmacol Toxicol Methods. 2012;65(1):18-28.

15. Sankaranarayanan M, Chua LP, Ghista DN, Tan YS. Computational model of blood flow in the aorto-coronary bypass graft. Biomed Eng Online. 2005:4:14.

16. Wendell DC, Samyn MM, Cava JR, Ellwein LM, Krolikowski MM, Gandy KL, Pelech AN, Shadden SC, LaDisa JF Jr. Including aortic valve morphology in computational fluid dynamics simulations: initial findings and application to aortic coarctation. Med Eng Phys. 2013;35(6):723-35. 
17. Albert S, Balaban RS, Neufeld EB, Rossmann JS. Influence of the renal artery ostium flow diverter on hemodynamics and atherogenesis. J Biomech. 2014;47(7):1594-602.

18. Liu X, Peng C, Xia Y, Gao Z, Xu P, Wang X, Xian Z, Yin Y, Jiao L, Wang D, et al. Hemodynamics analysis of the serial stenotic coronary arteries. Biomed Eng Online. 2017;16(1):127.

19. Wong KK, Thavornpattanapong P, Cheung SC, Tu J. Numerical stability of partitioned approach in fluid-structure interaction for a deformable thin-walled vessel. Comput Math Methods Med. 2013;2013:638519.

20. Siguenza J, Pott D, Mendez S, Sonntag SJ, Kaufmann TAS, Steinseifer U, Nicoud F. Fluid-structure interaction of a pulsatile flow with an aortic valve model: a combined experimental and numerical study. Int J Numer Method Biomed Eng. 2017. https://doi.org/10.1002/cnm.2945.

21. Sharzehee M, Khalafvand SS, Han HC. Fluid-structure interaction modeling of aneurysmal arteries under steadystate and pulsatile blood flow: a stability analysis. Comput Methods Biomech Biomed Eng. 2018;21:219.

22. Kim HJ, Vignon-Clementel IE, Figueroa CA, LaDisa JF, Jansen KE, Feinstein JA, Taylor CA. On coupling a lumped parameter heart model and a three-dimensional finite element aorta model. Ann Biomed Eng. 2009;37(11):2153-69.

23. Liu X, Pu F, Fan Y, Deng X, Li D, Li S. A numerical study on the flow of blood and the transport of LDL in the human aorta: the physiological significance of the helical flow in the aortic arch. Am J Physiol Heart Circ Physiol. 2009;297(1):H163-70.

24. LaDisa JF, Alberto Figueroa C, Vignon-Clementel IE, Jin Kim H, Xiao N, Ellwein LM, Chan FP, Feinstein JA, Taylor CA. Computational simulations for aortic coarctation: representative results from a sampling of patients. J Biomech Eng. 2011;133(9):091008

25. Liu X, Zhang H, Ren L, Xiong H, Gao Z, Xu P, Huang W, Wu W. Functional assessment of the stenotic carotid artery by CFD-based pressure gradient evaluation. Am J Physiol Heart Circ Physiol. 2016;311(3):H645-53.

26. Yang Y, Liu X, Xia Y, Liu X, Wu W, Xiong H, Zhang H, Xu L, Wong KKL, Ouyang H, Huang W. Impact of spatial characteristics in the left stenotic coronary artery on the hemodynamics and visualization of $3 \mathrm{D}$ replica models. Sci Rep. 2017;7(1):15452.

27. Xu P, Liu X, Song Q, Chen G, Wang D, Zhang H, Yan L, Liu D, Huang W. Patient-specific structural effects on hemodynamics in the ischemic lower limb artery. Sci Rep. 2016;6:39225.

28. Nash RW, Carver HB, Bernabeu MO, Hetherington J, Groen D, Kruger T, Coveney PV. Choice of boundary condition for lattice-Boltzmann simulation of moderate-Reynolds-number flow in complex domains. Phys Rev E Stat Nonlin Soft Matter Phys. 2014;89(2):023303.

29. Boyd J, Buick JM, Cosgrove JA, Stansell P. Application of the lattice Boltzmann method to arterial flow simulation: investigation of boundary conditions for complex arterial geometries. Australas Phys Eng Sci Med. 2004;27(4):207-12.

30. Chiu JJ, Chien S. Effects of disturbed flow on vascular endothelium: pathophysiological basis and clinical perspectives. Physiol Rev. 2011;91(1):327-87.

31. Wentzel JJ, Corti R, Fayad ZA, Wisdom P, Macaluso F, Winkelman MO, Fuster V, Badimon JJ. Does shear stress modulate both plaque progression and regression in the thoracic aorta? Human study using serial magnetic resonance imaging. J Am Coll Cardiol. 2005;45(6):846-54.

32. Dolan JM, Kolega J, Meng H. High wall shear stress and spatial gradients in vascular pathology: a review. Ann Biomed Eng. 2013;41(7):1411-27.

33. Thubrikar MJ, Agali P, Robicsek F. Wall stress as a possible mechanism for the development of transverse intimal tears in aortic dissections. J Med Eng Technol. 1999;23(4):127-34.

34. Wen CY, Yang AS, Tseng LY, Chai JW. Investigation of pulsatile flowfield in healthy thoracic aorta models. Ann Biomed Eng. 2010;38(2):391-402.

35. Nordon IM, Hinchliffe RJ, Loftus IM, Morgan RA, Thompson MM. Management of acute aortic syndrome and chronic aortic dissection. Cardiovasc Intervent Radiol. 2011;34(5):890-902.

36. LaDisa JF Jr, Dholakia RJ, Figueroa CA, Vignon-Clementel IE, Chan FP, Samyn MM, Cava JR, Taylor CA, Feinstein JA. Computational simulations demonstrate altered wall shear stress in aortic coarctation patients treated by resection with end-to-end anastomosis. Congenit Heart Dis. 2011;6(5):432-43.

37. Zhang $Q$, Gao B, Chang Y. The study on hemodynamic effect of series type LVAD on aortic blood flow pattern: a primary numerical study. Biomed Eng Online. 2016;15(Suppl 2):163.

38. Wong KK, Wang D, Ko JK, Mazumdar J, Le TT, Ghista D. Computational medical imaging and hemodynamics framework for functional analysis and assessment of cardiovascular structures. Biomed Eng Online. 2017;16(1):35.

39. Liu X, Fan Y, Deng X. Effect of spiral flow on the transport of oxygen in the aorta: a numerical study. Ann Biomed Eng. 2010;38(3):917-26.

40. Caro CG, Cheshire NJ, Watkins N. Preliminary comparative study of small amplitude helical and conventional ePTFE arteriovenous shunts in pigs. J R Soc Interface. 2005;2(3):261-6.

41. Wang Z, Liu X, Kang H, Sun A, Fan Y, Deng X. Enhanced accumulation of LDLs within the venous graft wall induced by elevated filtration rate may account for its accelerated atherogenesis. Atherosclerosis. 2014;236(1):198-206.

42. Mirzaee H, Henn T, Krause MJ, Goubergrits L, Schumann C, Neugebauer M, Kuehne T, Preusser T, Hennemuth A. MRI-based computational hemodynamics in patients with aortic coarctation using the lattice Boltzmann methods: clinical validation study. J Magn Reson Imaging. 2017;45(1):139-46.

43. Kwon S, Feinstein JA, Dholakia RJ, LaDisa JF. Quantification of local hemodynamic alterations caused by virtual implantation of three commercially available stents for the treatment of aortic coarctation. Pediatr Cardiol. 2013;35(4):732-40.

44. Dwyer HA, Matthews PB, Azadani A, Jaussaud N, Ge L, Guy TS, Tseng EE. Computational fluid dynamics simulation of transcatheter aortic valve degeneration. Interact Cardiovasc Thorac Surg. 2009:9(2):301-8.

45. Kilner PJ, Yang GZ, Mohiaddin RH, Firmin DN, Longmore DB. Helical and retrograde secondary flow patterns in the aortic arch studied by three-directional magnetic resonance velocity mapping. Circulation. 1993;88(5 Pt 1):2235-47. 